\title{
The Shape Distribution of Kuiper Belt Objects
}

\author{
Jane Luu \\ MIT Lincoln Laboratory \\ Pedro Lacerda \\ Leiden Observatory, University of Leiden
}

June 23, 2003

\begin{abstract}
If we assume that the periodic brightness variations in a Kuiper Belt lightcurve are determined only by their aspherical shapes and the observing geometry, the fraction of detectable Kuiper Belt lightcurves and the lightcurve amplitude distribution can be used to constrain the shapes of Kuiper Belt objects. The results indicate that most Kuiper Belt objects $(\sim 85 \%)$ have shapes that are close to spherical $(a / b \leq 1.5)$, but there is a small but significant fraction $(\sim 12 \%)$ possessing highly aspherical shapes $(a / b \geq 1.7)$. The distribution cannot be well fitted by a gaussian and is better approximated by a power law.
\end{abstract}

Keywords: Kuiper Belt objects, shape distribution

\section{Introduction}

Since their discovery in 1992, the Kuiper Belt objects (KBOs) have attracted a great deal of interest in planetary astronomy because of the information they might contain. Thought to be a relic from the original protoplanetary disk, they are expected to still bear signatures of their origin and evolution. In particular, they are believed to be much less evolved than other known solar system objects, and thus might show planetary formation at an early stage.

Although it has been a decade since their discovery, not much is known about the KBOs physical properties, mainly because most are too faint (red magnitude $m_{R} \geq 20$ ) for detailed studies. Most of the existing data are broadband photometry, with a few low-resolution optical and near-IR spectra. Broadband photometry indicates that the KBOs possess diverse colors, ranging from neutral to very red (Luu \& Jewitt, 1996; Tegler \& Romanishin, 2000; Jewitt \& Luu, 2001). The low-resolution KBO spectra are usually featureless, although a few show weak $2 \mu \mathrm{m}$ water ice absorption (Brown, Cruikshank \& Pendleton, 1999; Jewitt \& Luu, 2001). Some broadband photometric data have been obtained for the purpose of studying KBO rotational properties, and although reliable lightcurves are still relatively rare, the sample is sufficient for detailed analysis. In this paper we collect data

(C) 2003 Kluwer Academic Publishers. Printed in the Netherlands. 
from reliable lightcurves and examine their implications for the shape distribution of KBOs.

\section{Observations}

The largest and most systematic studies of KBO rotational properties were carried out by Sheppard \& Jewitt (2002, hereafter SJ02) and Sheppard \& Jewitt (2003, hereafter SJ03), which together present optical lightcurves of $27 \mathrm{KBOs}$. Their sample included most of the largest and brightest KBOs, with red absolute magnitude $H_{R}$ in the range $6.0-7.5$, corresponding to the diameter range $200-400 \mathrm{~km}$. These large objects are unlikely to be collisional fragments. Interestingly, only 7 of the 27 yielded periodic lightcurves, which they defined as periodic brightness variations with amplitude $\Delta m \geq 0.15$. Other lightcurves exist besides those presented by SJ02 and SJ03, but they vary widely in quality and sometimes contradict each other. For example, Romanishin \& Tegler (1999) report a flat lightcurve for 1996 TO66, while Hainaut et al. (2000) and SJ03 detected periodic lightcurve for the same object. Of the lightcurves in the literature that were not measured by them, SJ02 deemed 6 to be reliable and included them in their analysis. Of these 6 , 2 showed periodic lightcurves. In this work we adopt the same practice and make use of the 27 lightcurves from Sheppard \& Jewitt, plus the 6 reliable lightcurves from the literature. The KBO lightcurve statistics are thus as follows: out of 33 reliable lightcurves, 9 showed periodic modulations with amplitudes $\Delta m \geq 0.15$. The fraction of KBOs with detectable periodic lightcurves is then:

$$
f(\Delta m \geq 0.15)=9 / 33=27 \% .
$$

All 33 KBOs and their lightcurve parameters are listed in Table 1. Assuming that the lightcurves are modulated by aspherical shapes and are therefore double-peaked, the periods range from 6 to $12 \mathrm{hrs}$.

\section{Discussion}

Lacerda \& Luu (2003) show that the fraction of detectable KBO lightcurves can be used to infer these objects shape distribution, if certain (reasonable) assumptions are made. The assumptions are

1. Asphericity. The lightcurve modulations are assumed to arise from an aspherical shape, taken to be a triaxial ellipsoid with axes $a \geq$ $b \geq c$. The axis ratio $a / b$ must be large enough to give rise to 
a lightcurve amplitude $\Delta m$ which is greater than some minimum amplitude $\Delta m_{\min }$ set by the photometric error. In other words, $\Delta m=2.5 \log (a / b) \geq \Delta m_{\text {min }}$.

2. Observation geometry. The angle between the KBO spin axis and the line of sight - the aspect angle $\theta$ - should also be large enough to give rise to a detectable lightcurve amplitude, i.e., larger than $\Delta m_{\min }$.

Here we adopt SJ02's photometric error of 0.15 mag, i.e., $\Delta m_{\min }=$ 0.15. If a lightcurve does not show periodic modulations, it is assumed that this is because one of the two assumptions is not satisfied. No spin rate bias is considered here.

With these assumptions, the probability $p$ of detecting a lightcurve can be written as (Lacerda and Luu, 2003)

$$
p\left(\Delta m>\Delta m_{\min }\right)=\int_{0}^{1} \int_{1}^{\infty} \Psi(\bar{a}, \bar{c}) f(\bar{a}) g(\bar{c}) d \bar{a} d \bar{c} .
$$

where, for the sake of being concise, we define $\bar{a}=a / b, \bar{c}=c / b$, and $\Psi(\bar{a}, \bar{c})$ is the probability of detecting brightness variations from a given ellipsoid of axis ratio $(\bar{a}, \bar{c}) . \Psi(\bar{a}, \bar{c})$ is given by

$$
\Psi(\bar{a}, \bar{c})=\sqrt{\frac{\bar{c}^{2}\left(\bar{a}^{2}-K\right)}{\bar{c}^{2}\left(\bar{a}^{2}-K\right)+\bar{a}^{2}(K-1)}},
$$

where $K=10^{0.8 \Delta m_{\min }}$. The right hand side of Eqn. (2) represents the probability of observing a given $\mathrm{KBO}$ with axis ratios between $(\bar{a}, \bar{c})$ and $(\bar{a}+d \bar{a}, \bar{c}+d \bar{c})$ at a large enough aspect angle, integrated over all possible axis ratios. For moderately elongated ellipsoids (small $\bar{a}$ ), the function $\Psi(\bar{a}, \bar{c})$ is almost independent of $\bar{c}$. If we further assume $\bar{c} \approx$ $1, g(\bar{c})$ is $\approx 1$, and Eqn. (2) becomes

$$
p\left(\Delta m>\Delta m_{\min }\right) \approx \int_{1}^{\infty} \Psi(\bar{a}, 1) f(\bar{a}) d \bar{a} .
$$

From Eqn. (1), $p\left(\Delta m>\Delta m_{m i n}\right)=0.27$. Since the data did not sample the entire KBO population, there are necessarily errors associated with $p$. The 1-, 2- and $3 \sigma$ error bars on $p$ can be calculated based on the Clopper-Pearson confidence limits (Lacerda and Luu, 2003):

$$
\begin{array}{ll}
1 \sigma & p=0.27_{-0.08}^{+0.10} \\
2 \sigma & p=0.27_{-0.14}^{+0.19} \\
3 \sigma & p=0.27_{-0.18}^{+0.28}
\end{array}
$$


With $p$ known, the problem then becomes inverting Eqn. (4) to determine the shape distribution $f(\bar{a})$. This can be done if $f(\bar{a})$ is assumed to be a simple analytical function.

\subsection{Gaussian Distribution}

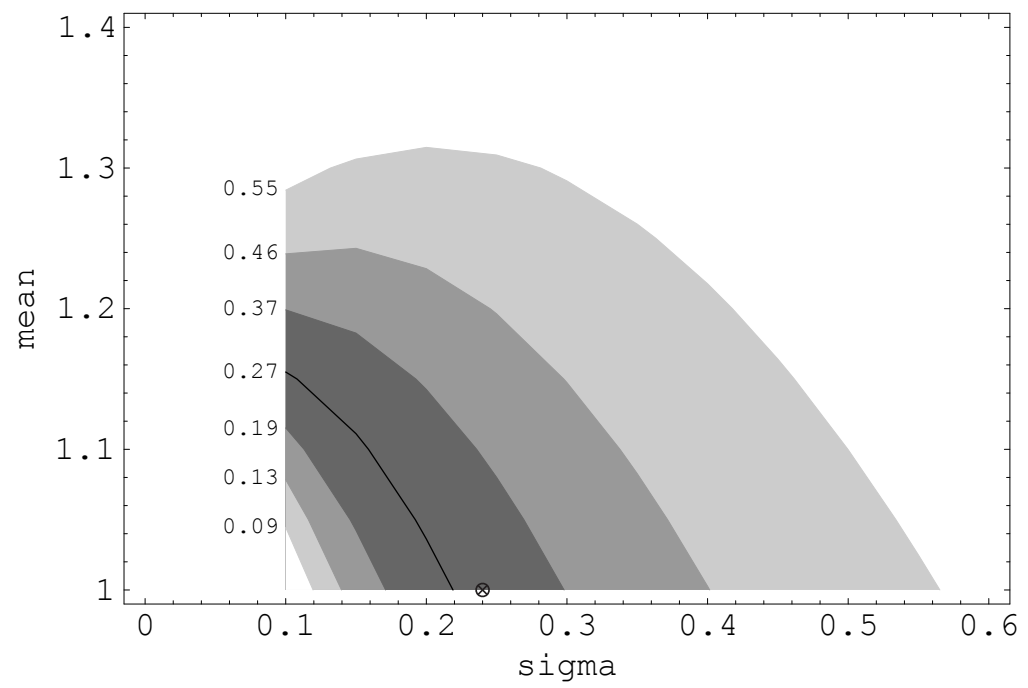

Figure 1. The probability $p$ as a function of the mean $\mu$ and standard deviation $\sigma$ of a gaussian $\bar{a}$ distribution. The thick black line represents all $\mu-\sigma$ pairs that give rise to $p=0.27$. The dark shaded areas immediately adjacent to the line represent the $\mu$ and $\sigma$ values within the $1 \sigma$ limits, the next shaded areas outward represent the $2 \sigma$ limits, and the outermost shaded area the $3 \sigma$ limits. The number to the left of each boundary line indicates the $p$ value corresponding to that line. The circle with the cross marks the best-fit $\mu-\sigma: \mu=1.00, \sigma=0.24$.

First we assume $f(\bar{a})$ to be a gaussian with a mean denoted by $\mu$ and a standard deviation $\sigma$. All possible combinations of $\mu-\sigma$ that satisfy Eqn. (5) are plotted in Fig. 1. The thick black curve represents all possible combinations of $\mu-\sigma$ that give rise to $p=0.27$, and the shaded regions represent the $\mu-\sigma$ pairs allowed by $p$ 's 1-, 2-, and $3 \sigma$ error bars. The entire shaded regions thus represent all possible combinations of $\mu-\sigma$ that are consistent with $p=0.27_{-0.18}^{+0.28}$.

We can constrain $\mu-\sigma$ further by making use of the observed axis ratios $\bar{a}$. Using the data from Table 1 , for each detected lightcurve, the observed $\Delta m$ is converted to the axis ratio $\bar{a}$ by using the relation (Lacerda and Luu, 2003) 


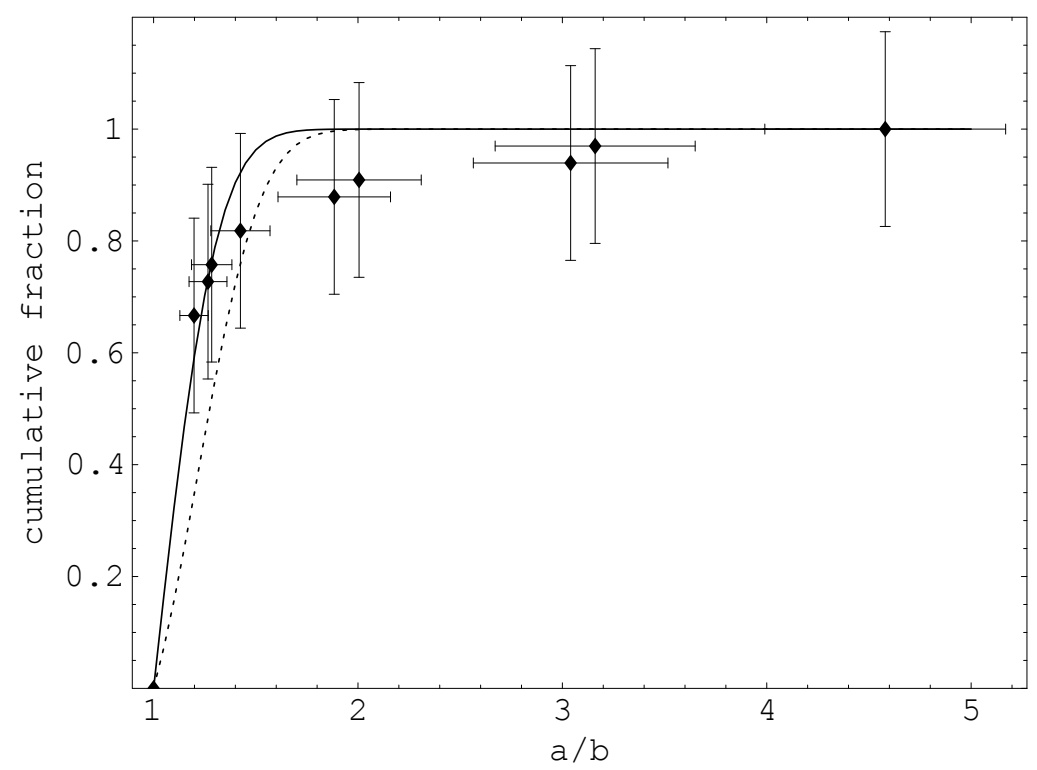

Figure 2. Cumulative fractions of KBOs as a function of $a / b(=\bar{a})$. The solid line corresponds to the best-fit gaussian $(\mu=1.00, \sigma=0.24)$, the dashed line a gaussian with $\mu=1.22, \sigma=0.24$. The data are from Table 1 , with vertical error bars calculated from Poisson statistics. The horizontal error bars are calculated from $1 \sigma$ deviation from the average aspect angle of $60^{\circ}$. Note: our $\mathrm{x}$-axis is $a / b$, which is the inverse of SJ02's x-axis, $b / a$.

$$
\Delta m=2.5 \log \left(\frac{\bar{a}^{2} \cos ^{2} \theta+\bar{a}^{2} \bar{c}^{2} \sin ^{2} \theta}{\bar{a}^{2} \cos ^{2} \theta+\bar{c}^{2} \sin ^{2} \theta}\right)^{1 / 2},
$$

and by assuming an average aspect angle $\theta=60^{\circ} \cdot\left(\theta=60^{\circ}\right.$ is the average angle if $\theta$ is distributed uniformly in $\sin \theta$ ). The observed cumulative fractions of KBOs, as a function of $a / b(=\bar{a})$, are plotted in Fig. 2. These can then be compared with the cumulative fractions predicted by each allowed $\mu-\sigma$ pair to yield the best-fit gaussian. A grid search is performed through all possible $\mu-\sigma$ pairs allowed by Eqn. (5); using $\chi^{2}$ as the comparison criterion, the best-fit gaussian is given by

$$
\mu=1.00(+0.22), \sigma=0.24_{-0.13}^{+0.18}
$$

The error bars in Eqn. (7) are $1 \sigma$ error bars. There is no lower error bar to $\mu$ since $\mu$ represents the mean $\bar{a}$, defined to be $\geq 1$. The best-fit $\mu-\sigma$ is also marked in Fig. 1. 
The goodness of the fit is shown graphically in Fig. 2 where we plot the observed cumulative fractions of KBOs, as a function of $\bar{a}$, with the cumulative fractions predicted by the best-fit gaussian (Eqn. 7). The fit is good for $\bar{a} \leq 1.5$ but poor at larger $\bar{a}$ 's. The Figure also shows that if we increase $\mu$ to $\mu=1.22$ ( 1 standard deviation away from the best-fit $\mu$ ), the theoretical curve comes closer to fitting the larger $\bar{a}$ 's, but misses practically all the data points.

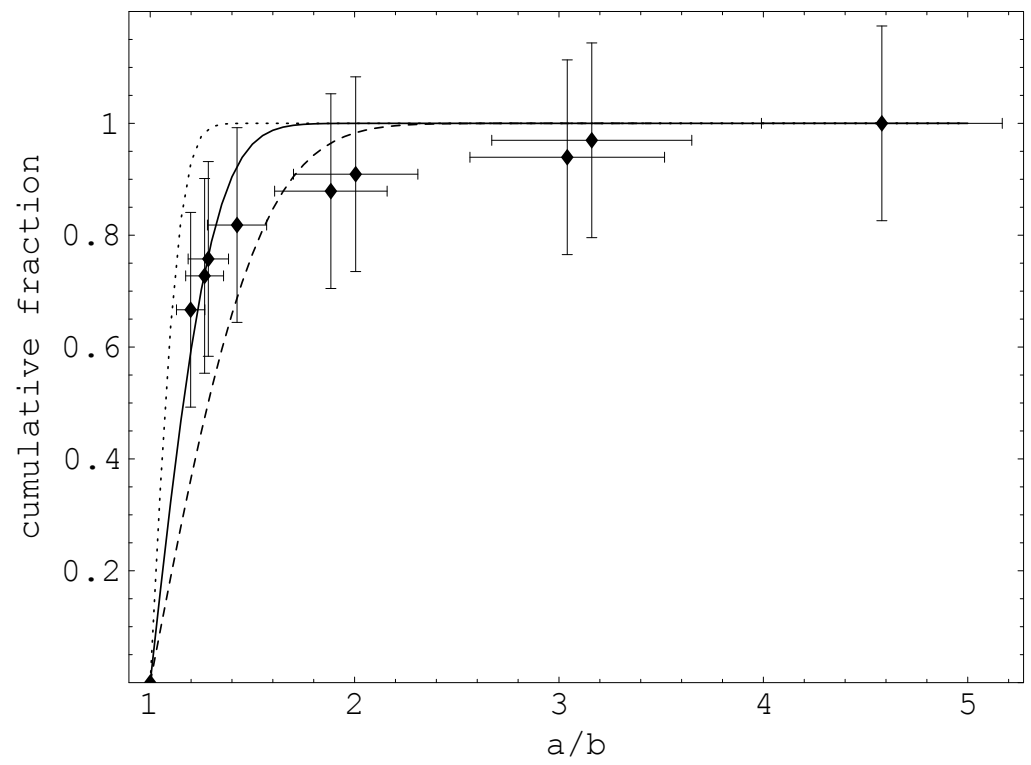

Figure 3. Cumulative fractions of KBOs as a function of $a / b(=\bar{a})$. Same as Fig. 2, but this time with the dotted line representing a gaussian with $\mu=1.00, \sigma=0.11$, the dashed line a gaussian with $\mu=1.00, \sigma=0.42$.

In Fig. 3 we try fitting the data with gaussians of different widths ( $\sigma=0.11$ and $\sigma=0.42$, both being 1 standard deviation away from the best-fit $\sigma$ ), while keeping $\mu$ fixed at $\mu=1.00$. The fit offered by $\sigma=0.11$ is much poorer than those seen in Fig. $2 ; \sigma=0.42$ comes closer to fitting all the data, but still misses all the data points.

What can we infer from Figs. 2 and 3? The best-fit gaussian (solid line in Fig. 2) is skewed toward small axis ratios and predicts that $95 \%$ of KBOs have axis ratios $a / b \leq 1.48$ (within $2 \sigma$ from the mean). This agrees reasonably well with the data which indicate that $\sim 85 \%$ have axis ratios $a / b \leq 1.48$. However, the best-fit gaussian fails badly at larger $a / b$ 's: it predicts that only $0.3 \%$ of KBOs have axis ratios $a / b \geq 1.72$ (larger than $3 \sigma$ from the mean), while the data indicate that $\sim 12 \%$ have axis ratios in this range. Increasing $\mu$ to $\mu=1.22$ 
(dashed line in Fig. 2) reduces some of this skewness but does not significantly improve the fit at larger $a / b$ 's.

Keeping $\mu$ fixed and decreasing $\sigma$ to $\sigma=0.11$ (dotted line in Fig. 3) worsens the fit, as expected. Keeping $\mu$ fixed and increasing to $\sigma=0.42$ (dashed line in Fig. 3) arguably produces the best fit yet (as judged by eye) since it tries to fit all the data points and does so equally well for all of them (or equally badly, depending on one's point of view). In short, none of the gaussians presented in Figs. 2 and 3 offers a good fit to the data. The conclusion to draw from the Figures is that the KBO shape distribution is not well approximated by a gaussian.

This is because the KBO shape distribution has two characteristics that cannot be met simultaneously by a standard gaussian: (1) a large fraction $(\sim 85 \%)$ has shapes that are close to spherical $(a / b \leq 1.5)$, yet $(2)$ there is a significant tail to the distribution $(\sim 12 \%)$ that has highly aspherical shapes $(a / b \geq 1.7)$. In other words, most KBOs are nearly spherical, but a signicant fraction is not. We note that, using a smaller data set, SJ02 came to the conclusion that a broad gaussian was needed to fit their available data. This is roughly consistent with our result here. With the benefit of a larger data sample, and the additional constraint from the detection probability $p$, we are able to improve SJ02's conclusion: the best description of the shape distribution is actually more like a moderately narrow peak with a long tail.

\subsection{Power LaW Distribution}

Considering how poorly a gaussian fits the shape distribution, we try approximating $f(\bar{a})$ with a power law, $f(\bar{a}) \propto \bar{a}^{q}$. The solution is shown graphically in Fig. 4, where the thick horizontal line represents $p=0.27$, and the solid black curve represents the detection probability $p$ as a function of $q$ :

$$
p(\bar{a}) d \bar{a}=\bar{a}^{-q} d \bar{a} .
$$

In Eqn. (8), $p(\bar{a}) d \bar{a}$ is the fraction of a $\mathrm{KBO}$ with axis ratios in the range $\bar{a}$ to $\bar{a}+d \bar{a}$. The probability is normalized so that the integral of $p(\bar{a})$ from $\bar{a}=1$ to $\bar{a}=5$ is equal to 1 . The shaded areas represent all possible values of $p$ within its 1-, 2-, and $3 \sigma$ error bars, so the allowed values of $q$ are those that lie within these shaded areas. We note that the horizontal line and the curved line intersect at $q=6.7$.

As was done in the previous section, we use the observed lightcurve amplitude distribution to constrain $q$ further. We compare the observed cumulative fractions of KBOs, as a function of $\bar{a}$, with the cumulative fractions predicted by each possible $q$. A grid search is performed 


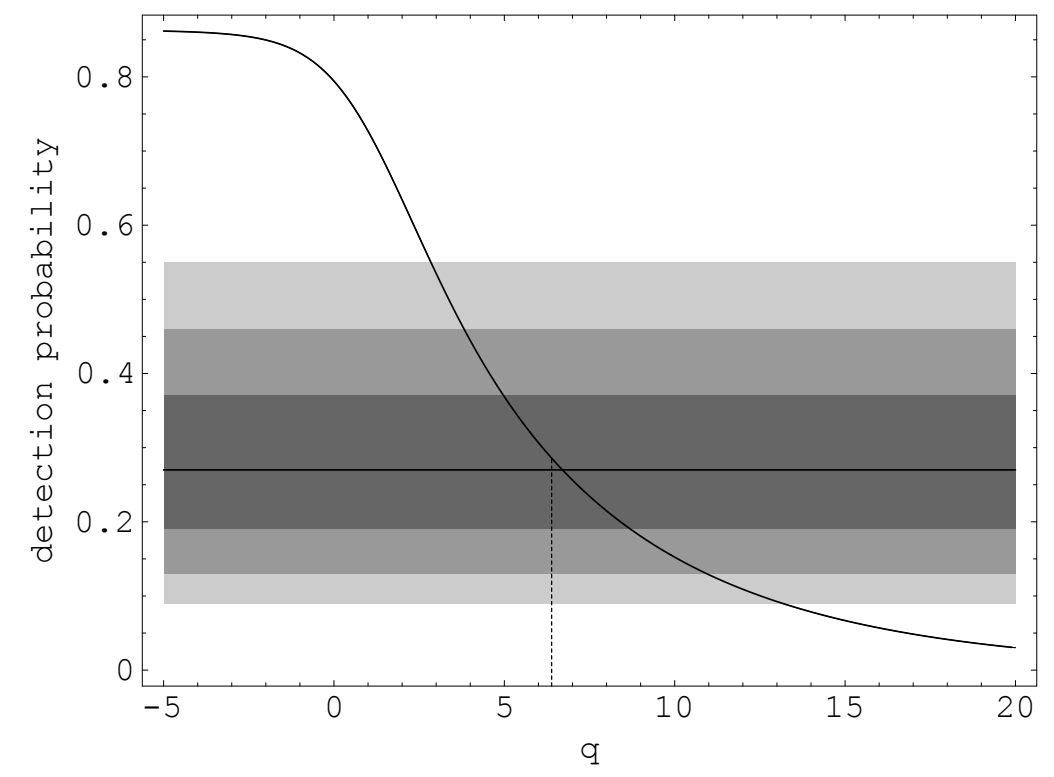

Figure 4. The detection probability $p$ as a function of the exponent $q$. The thick horizontal line represents all values of $q$ that that give rise to $p=0.27$. The shaded areas immediately adjacent to the line represent the values of $p$ within the $1 \sigma$ limits, the next shaded areas outward the $2 \sigma$ limits, and the outermost shaded area the $3 \sigma$ limits. The solid curve represents $p$ as a function of the exponent $q$ (Eqn. 8). The intersections of the shaded areas and the curve satisfy both Eqns. (2) and (8). The vertical line marks $q=6.4$.

through all possible values of $q$; using $\chi^{2}$ as the comparison criterion, we obtain the best-fit $q$ :

$$
q=6.4 \pm 1.4 \text { ( } 1 \sigma \text { error bars). }
$$

It is reassuring that $\chi^{2}$ statistics yield $q=6.4$ as the best fit, as this is very close to the $q=6.7$ found independently by the Lacerda \& Luu method. The fits offered by $q=5.0,6.4$ and 7.8 are shown in Fig. 5. It can be seen that the power laws generally fit the data better than the gaussians. We note, however, that the power laws still have the same problem as the gaussians: the fit is good at either the small $a / b$ data points or the large $a / b$ data points, there is no power law that fits all the data well. The shape distribution $f(\bar{a})$ as a gaussian and a power law is shown in Fig. 6.

As Fig. 6 shows, the KBO shape distribution is characterized by a steep peak at small $a / b$ 's, accompanied by a slow decline at larger $a / b$ 's. The dominance of small $a / b$ 's might be explained by (a) a preponderance of nearly spherical bodies, (b) a preponderance of very 


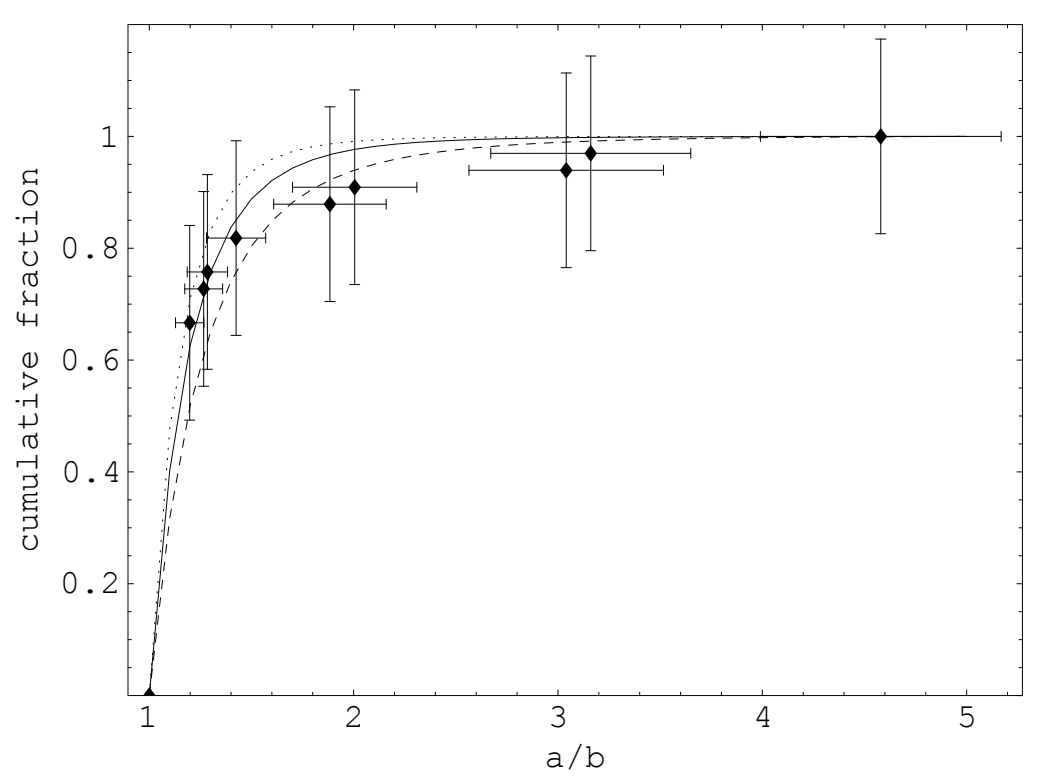

Figure 5. Cumulative fractions of KBOs as a function of $a / b(=\bar{a})$. Same as Fig. 2, but this time with the curves representing power laws. The dotted line represents the exponent $q=7.8$, solid line $q=6.4$, and dashed line $q=5.0$.

slow rotators whose lightcurve amplitudes could not be determined from the limited data, or some combination of these two factors. The likelihood of these scenarios will be evaluated in a future work. If the observational bias against slow rotators can be ruled out, the challenge will then be how to explain the dominance of nearly spherical bodies in the Kuiper Belt. As for the (small) fraction of KBOs with larger $a / b$ 's, Jewitt \& Sheppard (2002) and SJ02 have tentatively identified them as rotationally deformed "rubble piles," much like the "rubble piles" that have been proposed to exist among asteroids. If this hypothesis is correct, there should be a correlation between the KBO shapes and spin rates (high spin rate $\rightarrow$ large $\Delta m$ ). The data sample is as yet too small to confirm such a trend (e.g., see Fig. 13 of SJ02).

\section{Summary}

We have applied the method described in Lacerda \& Luu (2003) to the available KBO lightcurve data to constrain the KBO shape distribution. The method assumes that the detectability of KBO lightcurves depends only on the KBO shape and the observing geometry; it does 


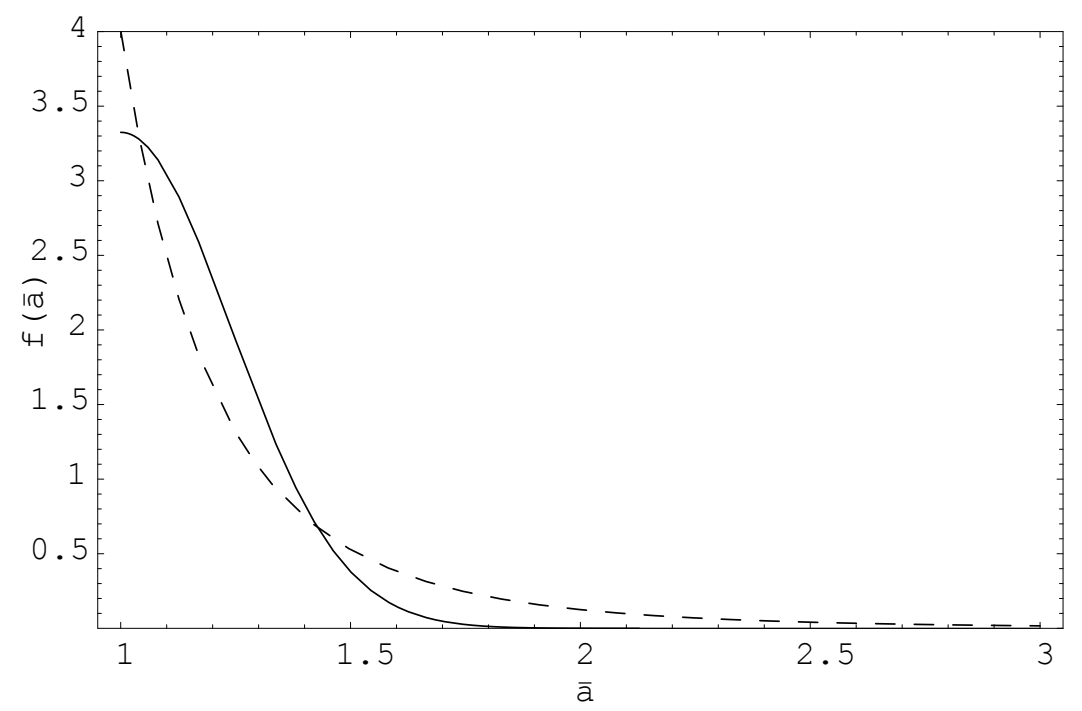

Figure 6. Distribution of axis ratio $\bar{a}$. The dashed line is the $q=6.4$ power law (Eqn. 9 ), the solid line a gaussian with $\mu=1.00, \sigma=0.24$ (Eqn. 7). $f(\bar{a})$ is normalized to 1 between $\bar{a}=1$ and $\bar{a}=5$.

not take into account any spin frequency effect (e.g., the bias against very slow spinners). The results can be summarized as follows:

1. With 9 out of 33 reliable KBO lightcurves showing periodic brightness variations, the fraction of detectable KBO lightcurves is $f(\Delta m \geq$ $0.15)=0.27$. This implies that the probability of detecting a KBO lightcurve is $p=0.27_{-0.18}^{+0.28}$ ( $3 \sigma$ error bars).

2. The KBO shape distribution has a steep peak at small axis ratios and drops off quickly to form a long tail: most of the distribution $(\sim 85 \%)$ has shapes that are close to spherical $(a / b \leq 1.5)$, yet (2) there is also a significant fraction $(\sim 12 \%)$ that has highly aspherical shapes $(a / b \geq 1.7)$.

3. Fitting the KBO $a / b$ distribution with a gaussian yields the best-fit mean $\mu=1.00(+0.22)$ and standard deviation $\sigma=0.24_{-0.13}^{+0.18}(1 \sigma$ error bars). However, this gaussian is strongly skewed toward small axis ratios $(a / b \leq 1.5)$, and offers a bad fit for larger axis ratios. Increasing the standard deviation reduces the skewness, but then all data points are fitted equally poorly. 
4. The KBO $a / b$ distribution is better fitted with power law distributions of the form $f(a / b) \sim(a / b)^{-q}$, with the best-fit exponent $q=6.4 \pm 1.4$ ( $1 \sigma$ error bars).

\section{Acknowledgements}

We thank Ronnie Hoogerwerf and Dave Jewitt for helpful discussion; we especially appreciate RH's expertise with $\mathrm{LT}_{\mathrm{E}} \mathrm{X}$ and SM. PL also thanks Scott Kenyon for a very fruitful and enjoyable visit to the Center for Astrophysics.

\section{References}

Brown, R. H., D. P. Cruikshank, \& Y. Pendleton. Water Ice on Kuiper Belt Object $1996 \mathrm{TO}_{66}$. ApJ, 519:L101-104, 1999.

Collander-Brown, S. J., A. Fitzsimmons, E. Fletcher, M. J. Irwin and I. P. Williams. Light curves of the trans-Neptunian objects $1996 \mathrm{TP}_{66}$ and $1994 \mathrm{VK}_{8} . M N R A S$, 308:588-592, 1999.

Davies, J. K., N. McBride and S. F. Green. Optical and Infrared Photometry of Kuiper Belt Object 1993 SC. Icarus, 125:61-66, 1997.

Green, S. F., N. McBride, D. P. O Ceallaigh, A. Fitzsimmons, I. P. Williams and M. J. Irwin. Surface Reflectance Properties of Distant Solar Dystem bodies. MNRAS, 290:186-192, 1997.

Hainaut, O. R. et al.. Lightcurves and Possible Cometary Activity. A\&A, 356:1076$1088,2000$.

Jewitt, D. C. and J. X. Luu. Colors and Spectra of Kuiper Belt Objects. AJ, 122:2099-2114, 2001.

Jewitt, D. C., and S. S. Sheppard. Physical Properties of Trans-Neptunian Object (20000) Varuna. AJ, 123:2110-2120, 2002.

Lacerda, P. and J. Luu. On the Detectability of Lightcurves of Kuiper Belt Objects. Icarus, 161:174-180, 2003.

Luu, J. X. and D. C. Jewitt. Color Diversity Among the Centaurs and Kuiper Belt Objects. AJ, 112:2310-2318, 1996.

Luu, J. X. and D. C. Jewitt. Optical and Infrared Reflectance Spectrum of Kuiper Belt Object 1996 TL$_{66}$. ApJ, 494:L117-120, 1998.

Ortiz, J. L., J. J. Lopez-Moreno, P. J. Gutierrez, and S. Baumont. A Study of Short Term Variability in $2001 \mathrm{KX}_{76}, 1999 \mathrm{TC}_{36}$ and $2000 \mathrm{QC}_{243}$. BAAS, 33:1047, 2001.

Romanishin, W. and S. C. Tegler. Rotation Rates of Kuiper Belt Objects from their Lightcurves. Nature, 398:129-132, 1999.

Romanishin, W., S. C. Tegler, T. W. Rettig, G. Consolmagno, and B. Botthof. (26308) 1998 SM165: A Large Kuiper Belt Object with an Irregular Shape. $B A A S, 33$ :abstract no. 06.06, 2001.

Sheppard, S. S. and D. C. Jewitt. Time-Resolved Photometry of Kuiper Belt Objects: Rotations, Shapes, and Phase Functions. AJ, 124:1757-1775, 2002. 
Sheppard, S. S. and D. C. Jewitt. Hawaii Kuiper Belt Variability Project: an Update. This volume, 2003.

Tegler, S. and W. Romanishin. Extremely red Kuiper-belt objects in near-circular orbits beyond 40 AU. Nature, 407:979-981, 2000. 
Table I. KBO rotational properties

\begin{tabular}{|c|c|c|c|}
\hline $\mathrm{KBO}$ & $P[\mathrm{hr}]$ & $\Delta m$ & Reference \\
\hline \multicolumn{4}{|c|}{ KBOs lightcurves considered to have $\Delta m<0.15 \mathrm{mag}$} \\
\hline $1993 \mathrm{SC}$ & - & 0.04 & RT99, DMcG97 \\
\hline $1994 \mathrm{~TB}$ & - & - & SJ02 \\
\hline 1996 GQ21 & - & $<0.10$ & SJ02 \\
\hline 1996 TL66 & - & 0.06 & RT99, LJ98 \\
\hline 1996 ТР66 & - & 0.12 & RT99, CB99 \\
\hline 1997 CS29 & - & $<0.08$ & SJ02 \\
\hline 1998 HK151 & - & $<0.15$ & SJ02 \\
\hline 1998 VG44 & - & $<0.10$ & SJ02 \\
\hline (Chaos) 1998 WH24 & - & $<0.10$ & SJ02 \\
\hline 1999 DE9 & - & $<0.10$ & SJ02 \\
\hline (47171) 1999 TC36 & - & $<0.10$ & SJ03 \\
\hline (Huya) 2000 EB173 & - & $<0.06$ & SJ02 \\
\hline 2000 YW134 & - & $<0.10$ & SJ03 \\
\hline $2001 \mathrm{CZ31}$ & - & $<0.20$ & SJ02 \\
\hline 2001 FP185 & - & $<0.10$ & SJ03 \\
\hline 2001 FZ173 & - & $<0.06$ & SJ02 \\
\hline $2001 \mathrm{KD} 77$ & - & $<0.10$ & SJ03 \\
\hline (28978) Ixion 2001 KX76 & - & $<0.10$ & SJ03,O01 \\
\hline 2001 QF298 & - & $<0.10$ & SJ03 \\
\hline (42301) 2001 UR163 & - & $<0.10$ & SJ03 \\
\hline (42355) 2002 CR46 & - & $<0.10$ & SJ03 \\
\hline (55636) 2002 ТX300 & $16.24 \pm 0.08$ & $0.08 \pm 0.02$ & SJ03 \\
\hline (55637) 2002 UX25 & - & $<0.10$ & SJ03 \\
\hline (55638) 2002 VE95 & - & $<0.10$ & SJ03 \\
\hline
\end{tabular}

KBOs lightcurves considered to have $\Delta m \geq 0.15 \mathrm{mag}$

$\begin{array}{lccl}\text { 1995 QY9 } & & 0.60 & \text { SJ02, RT99 } \\ \text { (24835) 1995 SM55 } & 8.08 \pm 0.03 & 0.19 \pm 0.05 & \text { SJ03 } \\ 1996 \text { TO66 } & - & 0.26 \pm 0.03 & \text { SJ03, H00 } \\ 1998 \text { SM165 } & & 0.45 & \text { SJ02, R01 } \\ 1998 \text { BU48 } & 9.8 \pm 0.1 & 0.68 \pm 0.04 & \text { SJ02 } \\ & 12.6 \pm 0.1 & & \\ 1999 \text { KR16 } & 11.858 \pm 0.002 & 0.18 \pm 0.04 & \text { SJ02 } \\ & 11.680 \pm 0.002 & & \\ \text { 2000 GN171 } & 8.329 \pm 0.005 & 0.61 \pm 0.03 & \text { SJ02 } \\ \text { (Varuna) 2000 WR106 } & 6.34 & 0.42 \pm 0.03 & \text { SJ02 } \\ \text { 2003 AZ84 } & 13.42 \pm 0.05 & 0.14 \pm 0.03 & \text { SJ03 }\end{array}$

CB99 = Collander-Brown et al. 1999, DMcG97 = Davies, McBride \& Green 1997, H00 = Hainaut et al. 2000, LJ98 = Luu \& Jewitt 1998, O01 = Ortiz et al. 2001, R01 $=$ Romanishin et al. 2001, RT99 = Romanishin \& Tegler 1999, SJ02 = Sheppard \& Jewitt 2002, SJ03 = Sheppard \& Jewitt 2003. 
KBOrotation2.tex; 23/06/2003; 13:26; p.14 